\title{
Anisotropy-Related Machining Characteristics in Ultra-Precision Diamond Cutting of Crystalline Copper
}

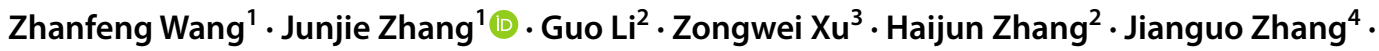 \\ Alexander Hartmaier ${ }^{5} \cdot$ Fengzhou Fang $^{3} \cdot$ Yongda Yan $^{1} \cdot$ Tao Sun $^{1}$
}

Received: 31 December 2019 / Revised: 24 February 2020 / Accepted: 20 March 2020 / Published online: 4 April 2020

(C) The Author(s) 2020

\begin{abstract}
Deformation behavior at grain levels greatly affects the machining characteristics of crystalline materials. In the present work, we investigate the influence of material anisotropy on ultra-precision diamond cutting of single crystalline and polycrystalline copper by experiments and crystal plasticity finite element simulations. Specifically, diamond turning and in situ SEM orthogonal cutting experiments are carried out to provide direct experimental evidence of the material anisotropy-dependent cutting results in terms of machined surface morphology and chip profile. Corresponding numerical simulations with the analysis of built stress further validate experimental results and reveal the mechanisms governing the material anisotropy influence. The above findings provide insight into the fabrication of ultra-smooth surfaces of polycrystalline metals by ultraprecision diamond turning.
\end{abstract}

Keywords Diamond cutting $\cdot$ Polycrystalline copper $\cdot$ Anisotropy $\cdot$ Grain boundary $\cdot$ Crystal plasticity finite element

\section{Introduction}

Ultimate surface finish with high surface integrity, low surface roughness, and high form accuracy is crucial for achieving superior optical, electrical, and chemical corrosion resistance properties of advanced components and parts [1-4]. Among different types of micro/nano-manufacturing techniques, mechanical machining such as ultra-precision

Junjie Zhang

zhjj505@gmail.com

1 Center for Precision Engineering, Harbin Institute of Technology, Harbin 150001, People's Republic of China

2 Research Center of Laser Fusion, China Academy of Engineering Physics, Mianyang 621900, People's Republic of China

3 State Key Laboratory of Precision Measuring Technology \& Instruments, Centre of MicroNano Manufacturing Technology, Tianjin University, Tianjin 300072, People's Republic of China

4 State Key Laboratory of Digital Manufacturing Equipment and Technology, School of Mechanical Science and Engineering, Huazhong University of Science and Technology, Wuhan 430074, People's Republic of China

5 Interdisciplinary Centre for Advanced Materials Simulation, Ruhr-University Bochum, 44780 Bochum, Germany single-point diamond turning (SPDT) with ultra-fine material removal has been demonstrated as a promising technique for achieving ultra-smooth surface of a variety of materials, such as metals [5-8], semiconductor materials [9-11], and ceramics [12-16]. Specifically, in addition to utilized precision CNC lathes and ultra-sharp diamond tools, SPDT is also highly dependent on the properties of workpiece materials, primarily due to the comparable feature size of internal microstructures of workpiece materials with depth of cut (DOC), as well as cutting edge radius of diamond tools. Thus, to facilitate the machining capability of SPDT, it is extremely important to evaluate the influence of material properties on the machining process.

In addition to atomic bonding forces that are used to categorize the classes of materials, international microstructures such as point defects, interfaces, and voids also have a strong impact on the properties of a specific material. For instance, polycrystalline metals are composed of multiple single crystalline grains with random grain sizes and random crystallographic orientations, as well as grain boundaries (GBs) that connect neighboring grains. While mechanical properties of individual grains with different crystallographic orientations are different from each other due to different the atomic packing sequences, mechanical responses of individual grains under external 
loading are also different. Specifically, the influence of material anisotropy on SPDT of metals, in terms of chip profile, machined surface morphology and cutting force, has been observed theoretically and experimentally. To et al. performed SPDT of single crystalline aluminum and found that the best surface finish is obtained with the $\{100\}$ plane, whereas the $\{110\}$ plane possesses the highest surface roughness [17]. Zhou et al. reported variations of cutting force and surface roughness with crystallographic orientation during SPDT of single crystalline aluminum and copper [18]. In particular for polycrystalline metals, Furukawa et al. concluded that the anisotropy of polycrystalline materials cannot be ignored in SPDT, as it may cause additional surface roughness around tens of nanometers [19]. Brinksmeier et al. found that since individual grains have different relative orientations and corresponding degrees of elastic and plastic recovery, surface steps with heights of a few nanometers may appear at GBs on the machined surface of polycrystalline copper under SPDT [20]. Yu et al. further demonstrated that the formation of GB surface step in SPDT of polycrystalline copper has a strong dependence on both the pre-treatment method of workpiece material and the grain size [21]. More recently, Wang et al. combined numerical simulation and experimental validation of orthogonal diamond cutting and scratching of bi-crystal copper, as well as cross-sectional TEM characterization, to demonstrate the formation mechanisms as well as suppression strategy of GB surface step on machined surface [22, 23].

To provide in-depth investigation of the mechanisms governing the material anisotropy-dependent diamond cutting processes, advanced experimental and numerical simulation techniques are needed. From the experimental side, in situ micro-cutting performed in a scanning electron microscope (SEM) could provide microscopic details of the on-going cutting process. Fang et al. performed in situ SEM micro-cutting experiments to successfully verify the transition of material removal from shearing to extrusion in diamond cutting with nanometer DOCs [24, 25]. Michler et al. embedded a microindentation/scratching apparatus into SEM to study the chip formation mechanisms during microscratching, and observe the phenomenon of material flow near the indenter [26]. Liu et al. specially designed nano-cutting platform embedded within SEM, and performed nano-cutting experiments of single crystalline silicon to study effects of crystal orientation and tool edge radius on the critical thickness for the brittle-to-ductile transition [27]. More recently, Wang et al. performed in situ SEM micro-cutting experiments of polycrystalline copper to examine the obtained chip profile, which are further verified by corresponding numerical simulations [28]. However, most of the previous in situ SEM micro-cutting experiments were focusing on the realization of cutting operations in SEM, and there is rare attention paid to the understanding of material properties' influence on the in situ cutting process.

From the numerical simulation side, finite element (FE) simulation has been widely used to investigate the cutting process, given its comparable length scale and time scale with experiments. However, the Johnson-Cook constitutive model adopted in most of the previous FE simulations of metal cutting cannot be used to address the influence of material anisotropy due to the simplification of material isotropy. While the crystal plasticity constitutive model (CPCM) has been widely used to describe deformation behavior of anisotropic materials under tension and compression tests with the absence of material removal, recently FE models incorporating CPCM have been established to address the anisotropy influence on the cutting of crystalline metallic materials. Lee et al. explored material anisotropy-induced variations of shear angle and cutting force in diamond cutting of polycrystalline copper by crystal plasticity finite element (CPFE) simulations [29]. Liu et al. performed CPFE simulations to successfully capture the influence of crystallographic orientation on the cutting force, chip morphology, and pile-up profile in cutting processes of single crystalline copper [30]. More recently, Wang et al. presents CPFE modeling and simulations of orthogonal diamond cutting of polycrystalline copper and corresponding experimental validation, in which the crystallographic orientations of individual grains between simulations and experiments are the same with electron backscatter diffraction (EBSD) characterization and corresponding Euler angles mapping [22, 28]. They also demonstrated a strong dependence of shearing deformation of single crystalline copper under orthogonal cutting on crystal anisotropy by CPFE simulations and experimental validation [31]. To facilitate the fundamental understanding of the material anisotropy influence on cutting processes of crystalline materials, further theoretical and experimental work is greatly needed.

In the present work, the influence of internal microstructure on orthogonal diamond cutting of both single crystalline and polycrystalline copper, in terms of chip profile, machined surface morphology, and cutting force, is investigated by experiments of diamond face turning and in situ SEM orthogonal cutting, as well as CPFE simulations of orthogonal cutting. Specifically, CPFE simulations are further performed to reveal the mechanisms governing the anisotropic machining characteristics in diamond cutting of polycrystalline copper.

\section{Methodology}

\subsection{Setup of Diamond Face Turning}

To demonstrate the impact of internal microstructure on diamond cutting, two types of workpiece materials were 
selected for direct comparisons, as (100)-oriented single crystalline copper and polycrystalline copper, respectively, which are obtained from Hefei Kejing Materials Technology Co., Ltd. The polycrystalline copper has an average grain size of $20 \mu \mathrm{m}$. As-received copper specimens are firstly subjected to vacuum annealing treatment at $800{ }^{\circ} \mathrm{C}$ for $1 \mathrm{~h}$,

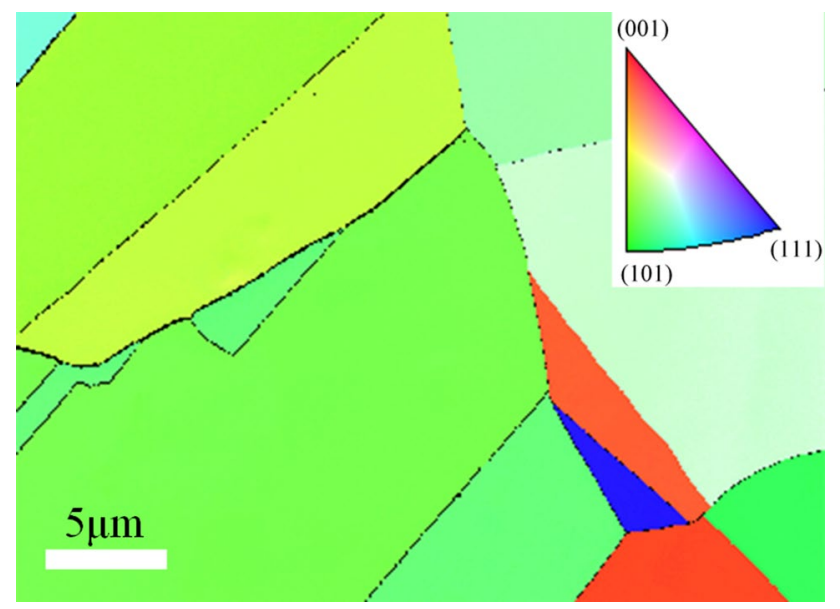

Fig. 1 EBSD image of characterized polycrystalline copper and then characterized by electron backscattered diffraction (EBSD) analysis to examine the misorientation and texture. Figure 1 shows the EBSD characterization of polycrystalline copper, which shows clearly individual grains and GBs.

Ultra-precision SPDT experiments were carried out on the Precitech Nanoform 700 precision CNC lathe, as shown in Fig. 2a. Two types of diamond tools were used. One is a round-nose diamond tool with a nose radius of $0.1 \mathrm{~mm}$ (shown in Fig. 2b), which is adopted for diamond face turning. Figure $2 \mathrm{c}$ shows the other diamond tool, which has a straight cutting edge of $1.2 \mathrm{~mm}$ in length and is used for orthogonal cutting. Due to the difficulty in tool position adjusting, there is an inclination of $0.2^{\circ}$ of the straight cutting edge with respect to the horizontal line. Each cutting tool has a rake angle of $0^{\circ}$, a clearance angle of $10^{\circ}$, and a tip radius of $60 \mathrm{~nm}$, respectively. In the diamond face turning using the round-nose diamond tool, the rotation speed of the spindle is $2000 \mathrm{rpm}$, the DOC is $0.5 \mu \mathrm{m}$, and the feed is $1.0 \mu \mathrm{m} / \mathrm{r}$. In the orthogonal cutting using the diamond tool with straight cutting edge, the DOC is $200 \mathrm{~nm}$ and the cutting speed is $30 \mathrm{~mm} / \mathrm{min}$. The machined surface morphology is measured with a white light interferometer and atomic force microscopy (AFM).
Fig. 2 Experimental setup of SPDT. a Configuration of tool clamping and workpiece fixture; b round-nose diamond tool; c diamond tool with straight cutting edge
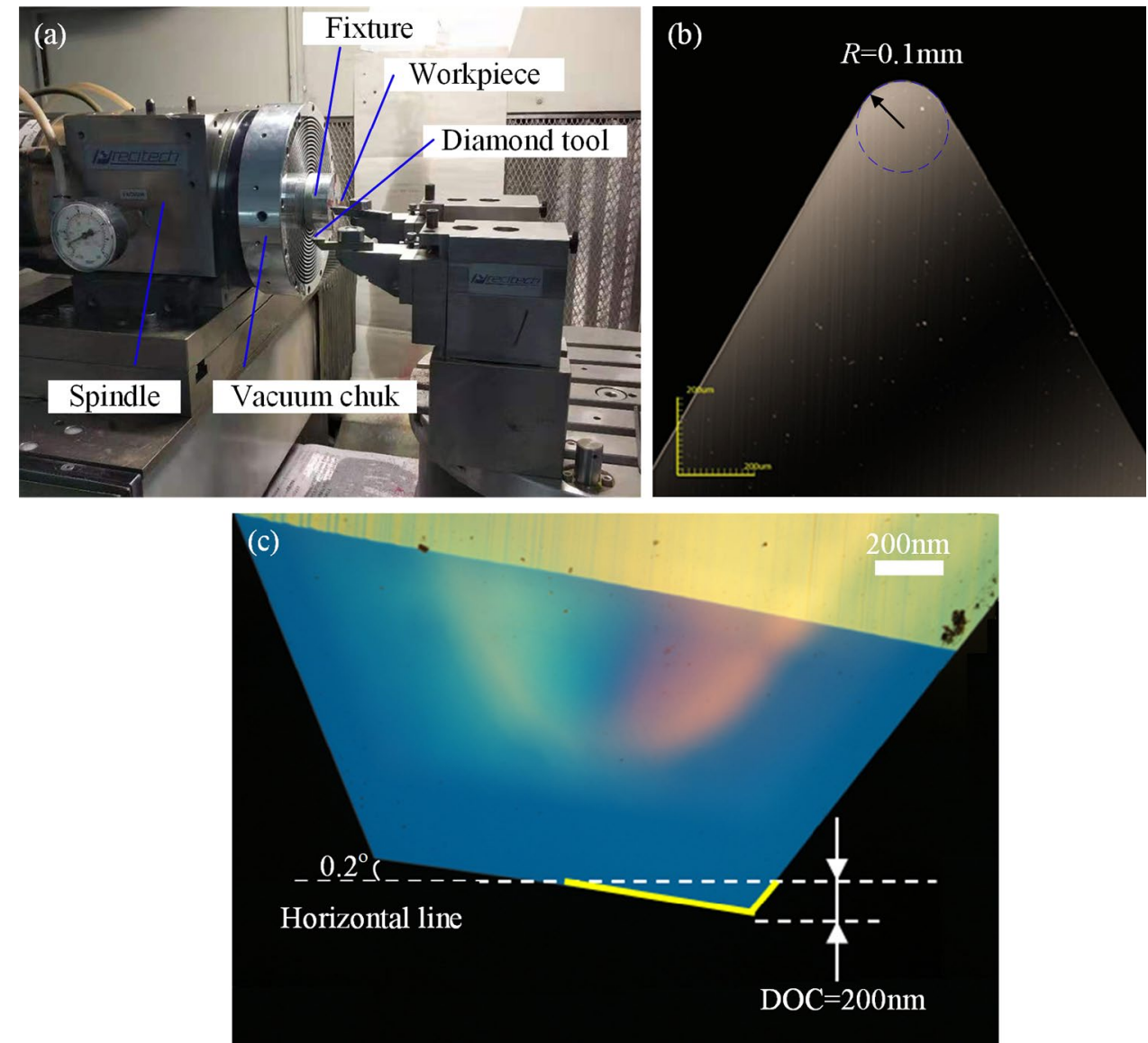


\subsection{Setup of In Situ SEM Cutting}

In situ micro-cutting experiments were performed by integrating a micro-cutting apparatus in SEM, where the detailed description can be found elsewhere [32]. A diamond tool with a straight cutting edge fabricated by focused ion beam is especially used for the orthogonal cutting. In the micro-cutting process, the tool is firstly penetrated into the specimen to achieve a pre-determined DOC of $200 \mathrm{~nm}$, and then cuts the specimen surface with a constant cutting speed of $1 \mu \mathrm{m} / \mathrm{s}$ until reaching a cutting distance of $6 \mu \mathrm{m}$.

\subsection{CPFE Modeling of Orthogonal Cutting of Crystalline Copper}

Figure 3a shows a 2D CPFE model of orthogonal cutting, which consists of a copper specimen and a diamond tool. The specimen has a dimension of $12 \mu \mathrm{m}$ in length and $5 \mu \mathrm{m}$ in width. The bottom and left sides of the specimen are completely fixed at space to prevent any rigid movement under the action of cutting force. Linear quadrilateral elements (CPE4) with an element size of $20 \mathrm{~nm}$ are adopted to mesh the specimen, which transforms the three-dimensional cutting with a straight cutting edge into a two-dimensional plain strain problem. The diamond tool is set as an analytical rigid body for its higher hardness than that of the copper specimen. In the cutting process, the diamond tool moves along the negative $X$ direction with a constant speed of $1 \mu \mathrm{m} / \mathrm{s}$ and a constant DOC of $200 \mathrm{~nm}$ until a cutting distance of $6 \mu \mathrm{m}$ is reached.

Figure $3 \mathrm{~b}$ presents the constructed polycrystalline copper specimen with random crystallographic orientations and random grain sizes with the Neper software according to the Voronoi algorithm [33]. The specimen consists of 12 individual grains of convex polyhedrons with an averaged grain size of $1.14 \mu \mathrm{m}$. While the utilized DOC in both experiments and CPFE simulations of cutting is the same as $200 \mathrm{~nm}$, a smaller grain size of $1.14 \mu \mathrm{m}$ in CPFE simulations than that of $20 \mu \mathrm{m}$ in experiments won't substantially affect the cutting behavior, and a small model can not only maintain high accuracy but also greatly improve computational efficiency, given the high computational cost of the implicit UMAT subroutine. To represent the crystal anisotropy, in the CPFE model the constructions of both single crystalline and polycrystalline copper specimens are based on the mapping of Euler angles derived from according EBSD characterization. The CPCM is adopted to describe the anisotropy of crystalline copper by developing a corresponding UMAT user subroutine in implicit analysis with the software ABAQUS $[28,34]$, and the shear strain rate can be expressed as a ratedependent power-law proposed by Pan et al. [35].

\section{Results and Discussion}

\subsection{Diamond Turning: Single Crystalline Versus Polycrystalline}

SPDT experiments of single crystalline and polycrystalline copper using a round-nose diamond tool were performed. Figure 4 presents machined surface morphologies of crystalline copper characterized with a white light interferometer. Figure 4 shows that the single crystalline copper specimen has a smaller surface roughness of $5.409 \mathrm{~nm}$ than that of $6.721 \mathrm{~nm}$ for the polycrystalline counterpart. Figure $4 \mathrm{a}$ shows that the machined surface of single crystalline copper has long-range uniform and consistent characteristics in both cutting and feed directions. Furthermore, the deviation of surface height fluctuating regularly in feed direction is below $\pm 10 \mathrm{~nm}$. In contrast, Fig. 4b shows that the fluctuation of machined surface height for the polycrystalline copper is more pronounced than that for the single crystalline counterpart. In spite of short-range continuous morphologies within individual grains, there is no long-range uniformity of surface finish observed.

Figure 5a further presents enlarged characterization of machined surface morphology in the vicinity of GB for polycrystalline copper by AFM, in which the border of GB is highlighted by a white dashed line. The similar AFM characterization of single crystalline copper is also presented in Fig. 5b for comparison purpose. In particular, Fig. 5a demonstrates that there is an obvious transition border of machined surface height on the GBs, and the fluctuation of surface height in grain interior is also different for each
Fig. 3 a 2D CPFE model of orthogonal cutting. b Grain configurations of polycrystalline copper
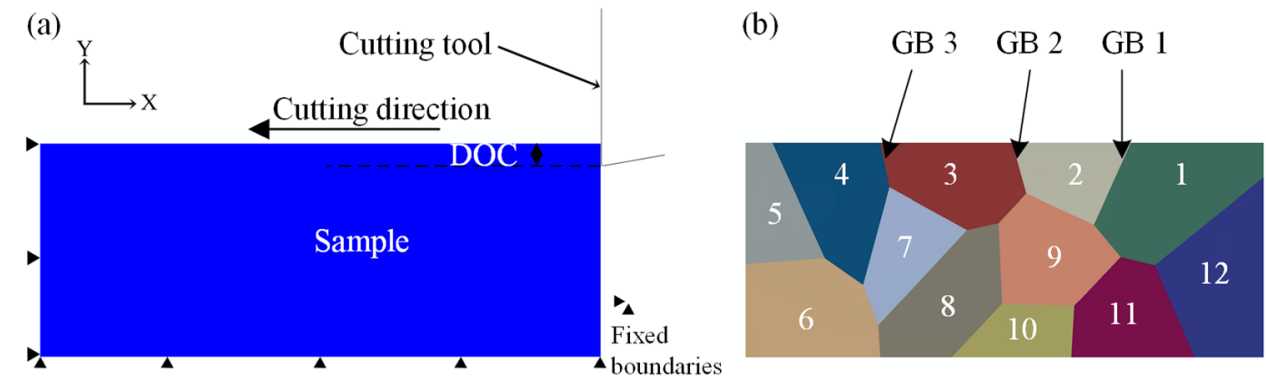

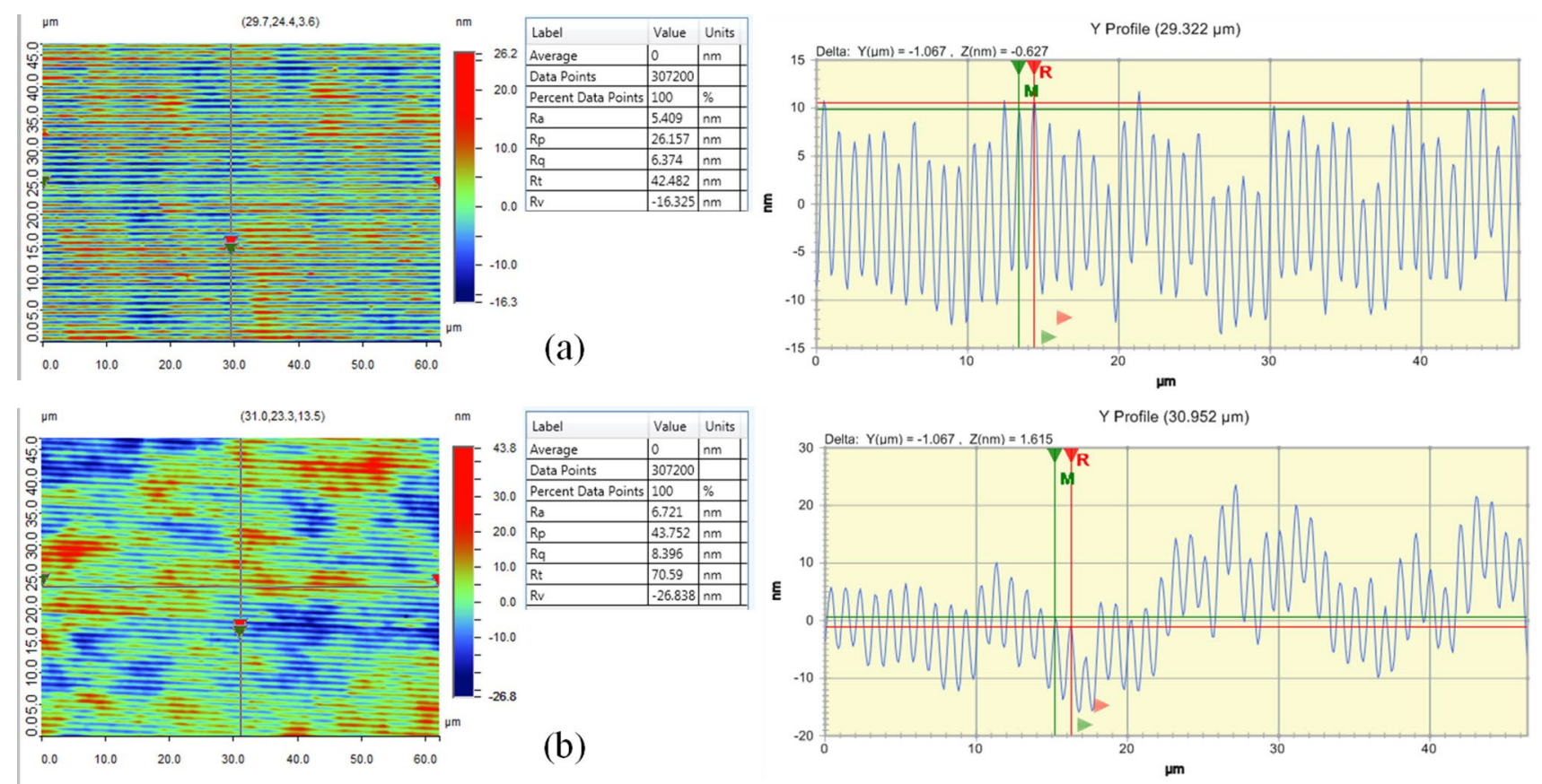

Fig. 4 Machined surface morphology measured by white light interferometer for a single crystalline and bolycrystalline copper specimen

Fig. 5 Machined surface morphology measured by AFM of a polycrystalline and $\mathbf{b}$ single crystalline copper
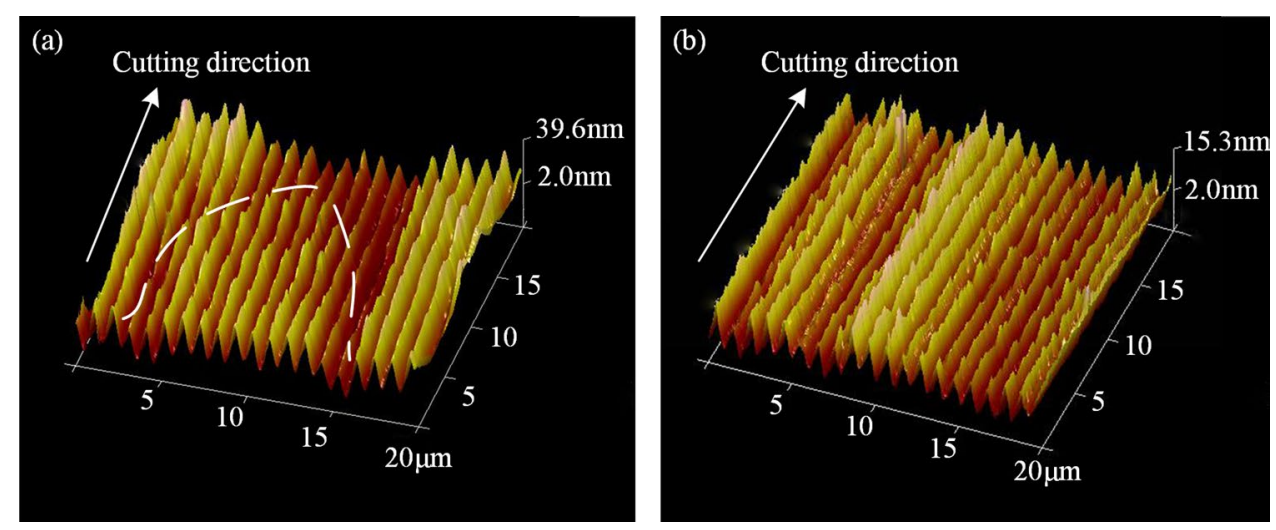

grain. However, a continuous and uniform distribution of surface height in cutting direction is observed for single crystalline copper, as indicated by Fig. 5b. The observed anisotropic characteristics of machined surface morphology for polycrystalline copper can be primarily attributed to different degrees of elastoplastic deformation and recovery in individual grains. The surface finish obtained during diamond machining depends on many factors, such as machining parameters, diamond tool geometry, precision lathe, etc. However, the GBs also have a strong impact on the ultimate surface finish.

In addition to diamond face turning, orthogonal diamond cutting experiments of single crystalline and polycrystalline copper using a diamond tool with straight cutting edge are also performed, with a particular interest on the effect of anisotropy on chip formation. Figure 6a, c shows an SEM image containing groove and chip in orthogonal diamond cutting under a DOC of $0.2 \mu \mathrm{m}$ for single crystalline and polycrystalline copper specimen, respectively. Because the diamond tool is inclined at $0.2^{\circ}$ relative to the parallel line due to the difficulty in tool position adjusting, the resultant groove geometry is V-shaped. Furthermore, Fig. 6b, d presents an SEM image of an enlarged view of the chip profile for a single crystalline and polycrystalline copper specimen, respectively. Figure 6 shows that both the surface finish and chip profile of single crystalline copper are significantly different from that of polycrystalline counterpart. Specifically, continuous chip profile is observed for single crystalline copper, and the chip-free surface is composed of regular lamellae, which indicate that the cutting process is stable. 

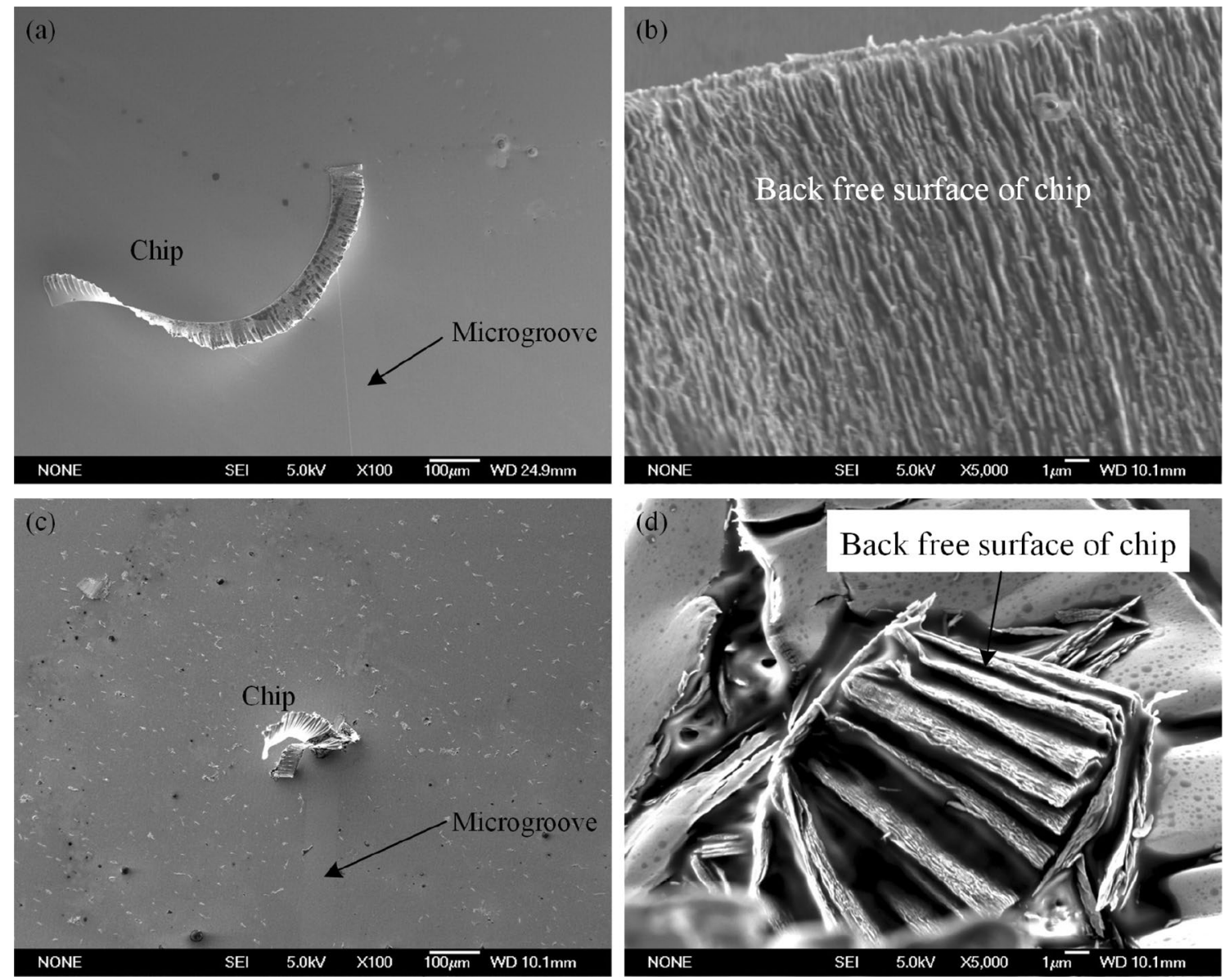

Fig. 6 SEM characterization of machined surface after orthogonal cutting. Machined surface morphology for $\mathbf{a}$ single crystalline and $\mathbf{b}$ polycrystalline copper; Enlarged view of chip profile for $\mathbf{c}$ single crystalline and $\mathbf{d}$ polycrystalline copper

In contrast, the length of continuous chip fragments is significantly decreased for polycrystalline copper, and there are pronounced serrations of complex lamella structures on the chip-free surface, which lead to easy folding of chip fragments. Furthermore, fractured debrises are also visible on the machined surface of polycrystalline copper. It is speculated that the more complex chip profile for polycrystalline copper than that for single crystalline counterpart is caused by serious confinement of plastic deformation within grain interiors by GBs. Thus, the surface finish is better for single crystalline copper than that for polycrystalline counterpart. In general, the existence of grains with different orientations and GBs jointly destroys the stability of cutting process, which in turn affect chip profile and machined surface morphology.

\subsection{In Situ Cutting: Single Crystalline Versus Polycrystalline}

Figure $7 \mathrm{a}, \mathrm{b}$ presents the workpiece-tool configuration after in situ SEM cutting with a cutting distance of $6 \mu \mathrm{m}$ for single crystalline and polycrystalline copper, respectively. It is seen from Fig. 7a that the chip profile of single crystalline copper is generally continuous and flat, indicating that the plastic deformation is dominated by uniform and tiny local shear slip events. However, the chip profile of polycrystalline copper consists of irregular serrated segmentations. As compared to the chip profile for single crystalline copper, the lamellar structure on the back chip-free surface becomes more complicated and the chip thickness is also larger for polycrystalline copper, which can be attributed to that the formed chips are contributed by different plastic deformation in adjacent grains with different crystallographic orientations.

\subsection{CPFE Simulation: Single Crystalline Versus Polycrystalline}

In addition to experimental investigations, CPFE simulations of orthogonal diamond cutting are also carried out to get in-depth insights into the anisotropy-dependent cutting of crystalline copper. Figure 8 shows CPFE simulation results 
Fig. 7 Chip profiles in in situ microcutting experiment of a single crystal copper and $\mathbf{b}$ polycrystalline copper
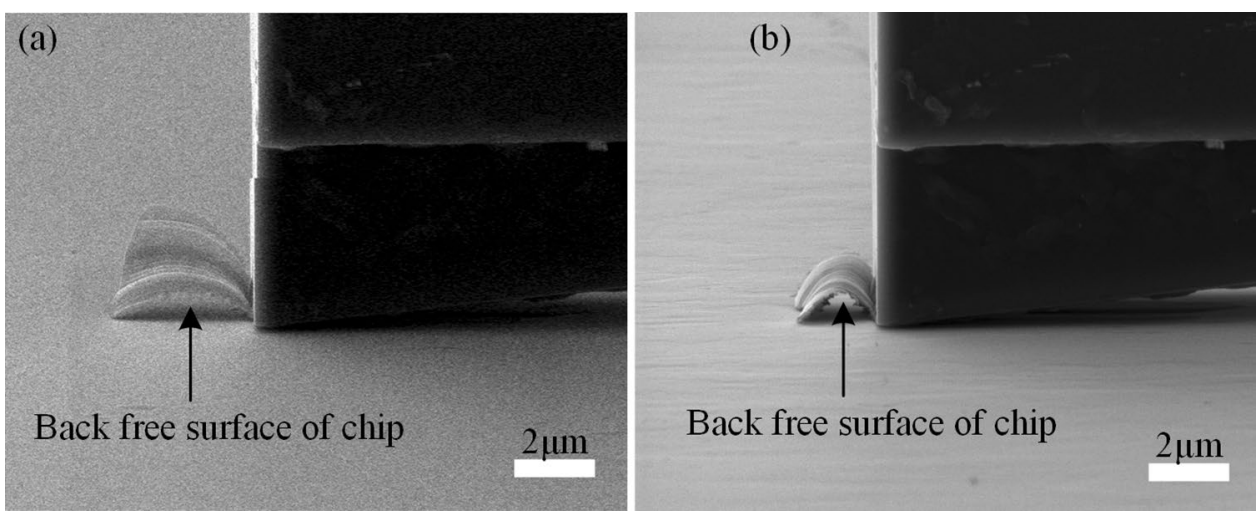

of deformation behavior of polycrystalline copper at different cutting distances, in which workpiece is colored according to both von Mises stress and critical resolved shear stress (CRSS).

Figure $8 \mathrm{a}, \mathrm{b}$ shows that the distributions of von Mises stress near the diamond tool and CRSS on formed chip for cutting in Grain 1 interior are very similar to that for single crystalline copper. This can be attributed to the blocking effect of GBs on the evolution of the stress accumulation by hindering propagation of dislocations from Grain 1 into Grain 2, which thus leads to stress concentration on the GBs. However, Fig. 8a indicates that GBs surrounding Grain 1 prevent further extension of von Mises stress at a certain distance from the diamond tool, which results in the confinement of stress distribution within Grain 1. As shown in Fig. 8c, the stress in front of the diamond tool expands along the GBs between Grain 3 and Grain 9 when the diamond tool is passing GB 1, and has the tendency to expand into the interiors of Grain 7 and Grain 8. Thus, it suggests that GBs act as both sinks for blocking dislocation motion and sources for dislocation nucleation. When the cutting is performed in Grain 2, Fig. 8e shows that new distribution pattern of von Mises stress is generated accompanied by the synergetic deformation between neighboring grains. At the same time,
Fig. 8 The distribution of von Mises stress (left row) and CRSS (right row) during cutting processes of polycrystalline copper at different cutting distances. Cutting distance: a, b $1.4 \mu \mathrm{m} ; \mathbf{c}, \mathbf{d} 3.2 \mu \mathrm{m} ; \mathbf{e}, \mathbf{f} 4.0 \mu \mathrm{m}$

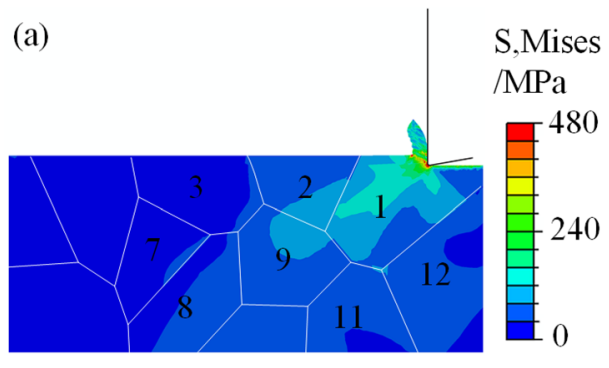

(c)

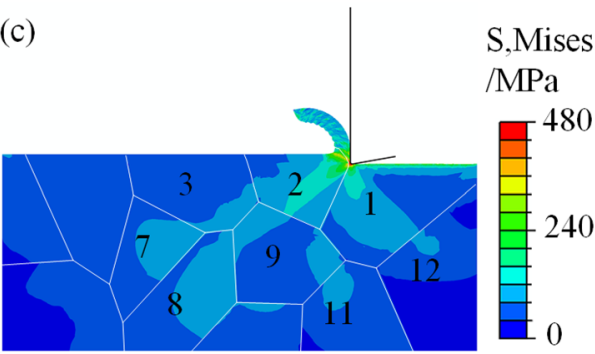

(e)

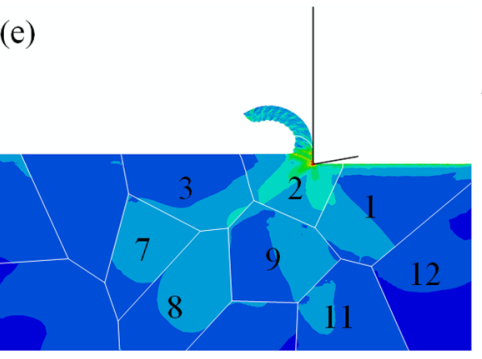

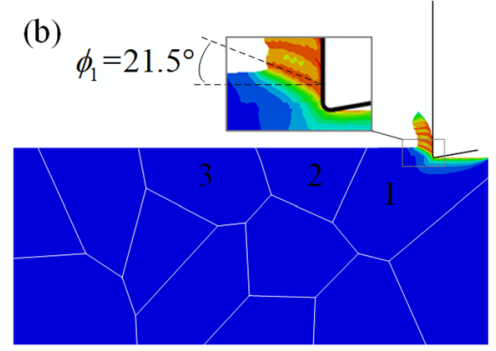

CRSS

\section{$/ \mathrm{MPa}$}

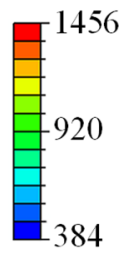

(d)

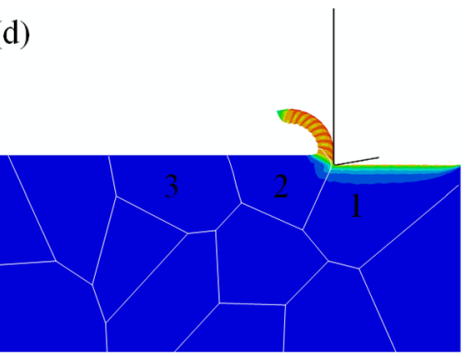

CRSS

$/ \mathrm{MPa}$
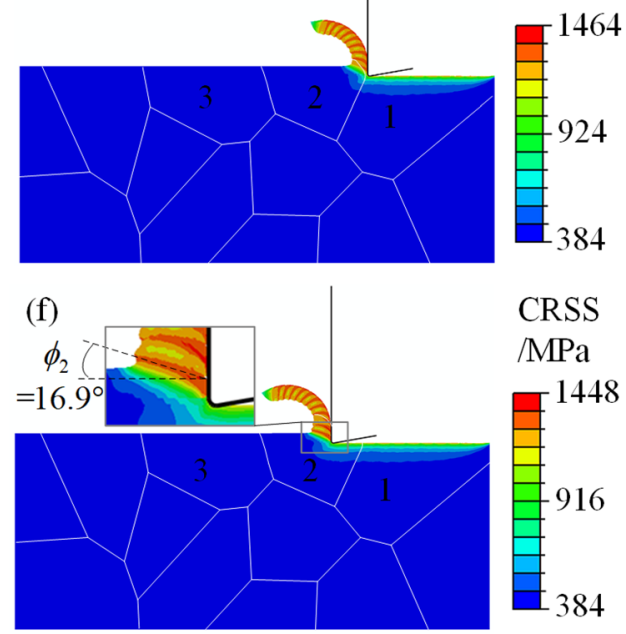
CRSS distribution shown in Fig. 8f also indicates different chip profiles between Grain 2 and Grain 1. As shown in Fig. 8a, b, the shear angle during the cutting process is variable with crystallographic orientation. Specifically, the value of shear angle is $21.5^{\circ}$ for Grain 1 and $16.9^{\circ}$ for Grain 2 . As a result, the formed chip in Grain 2 has a larger thickness than that in Grain 1.

Figure 9 shows distribution maps of both von Mises stress and CRSS of single crystalline copper and polycrystalline copper at the same cutting distance of $6 \mu \mathrm{m}$. It can be observed from Fig. 9a that for single crystalline copper, the maximum value of von Mises stress firstly occurs near the cutting edge, and then gradually decreases along two directions. While the material is mainly pressed by the tool feeding in direction 1 , in direction 2 the material affected by the ploughing effect forms the machined surface after the tool pass. As compared to single crystalline copper, the stress distribution in polycrystalline copper shown in Fig. 9b clearly demonstrates that grain structure has a significant effect on stress distribution pattern. Specifically, the extension of stress is blocked by GBs, which leads to significant stress concentration in the vicinity of GBs.

Figure 9c shows CRSS distribution in single crystalline copper, which indicates that severe plastic deformation in chip profile forms primary shear zone (PSZ) and regular chip lamellar. For the cutting process of polycrystalline copper, however, Fig. 9d shows that the neighboring Grain 1 and Grain 2 have totally different plastic deformation accompanied by different crystallographic orientations. The variation of PSZ with crystallographic orientation during chip formations indicates that complex chip lamellar occurs in diamond cutting of polycrystalline copper, which is consistent with experimental observation of chip profile in diamond cutting experiments.
Figure 10 plots variations of cutting force and surface height (i.e., height change from the initial position) with cutting distance in CPFE simulations of diamond cutting of single crystalline and polycrystalline copper. While Fig. 10a shows that cutting force for single crystalline copper fluctuates around a constant value of $85 \mathrm{mN}$ in stable cutting stage after initial rapid increase, Fig. 10c also demonstrates that the corresponding surface height changes regularly in the middle stage of the cutting process. However, when cutting is performing in polycrystalline copper, the variations of cutting force and surface height in Grain 1 and Grain 2 are dramatically different. Specifically, the cutting force in Grain 1 possesses more pronounced fluctuation events but around a smaller constant value than that in Grain 2. Furthermore, the fluctuation of surface height in Grain 1 is more pronounced but with a smaller fluctuation amplitude than that in Grain 2 . The significantly different machining characteristics between neighbor grains are attributed to grain anisotropy accompanied by different crystallographic orientations.

\section{Conclusions}

In summary, we perform experiments and CPFE simulations of ultra-precision diamond cutting of single crystalline and polycrystalline copper, aiming to reveal the impact of material anisotropy on the cutting processes. Experimental results of diamond face turning and orthogonal cutting, as well as in situ SEM orthogonal cutting, jointly demonstrate that both surface finish and chip profile of polycrystalline copper is significantly different from that of single crystalline counterpart. Specifically, while single crystalline copper possesses a long-range uniform surface finish, the machined surface morphology of polycrystalline copper exhibits a
Fig. 9 Distribution of von Mises stress for a single crystalline and $\mathbf{b}$ polycrystalline copper; Distribution of CRSS for c single crystalline and $\mathbf{d}$ polycrystalline copper

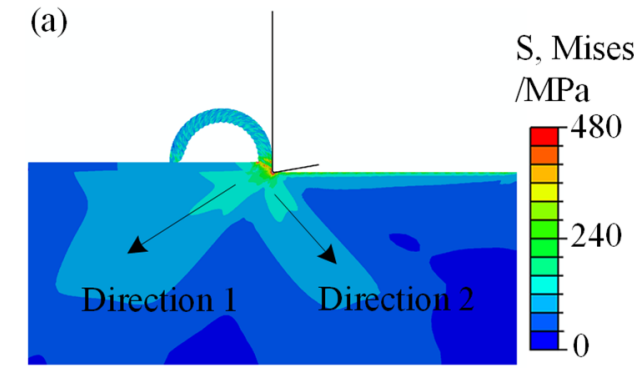

(b)
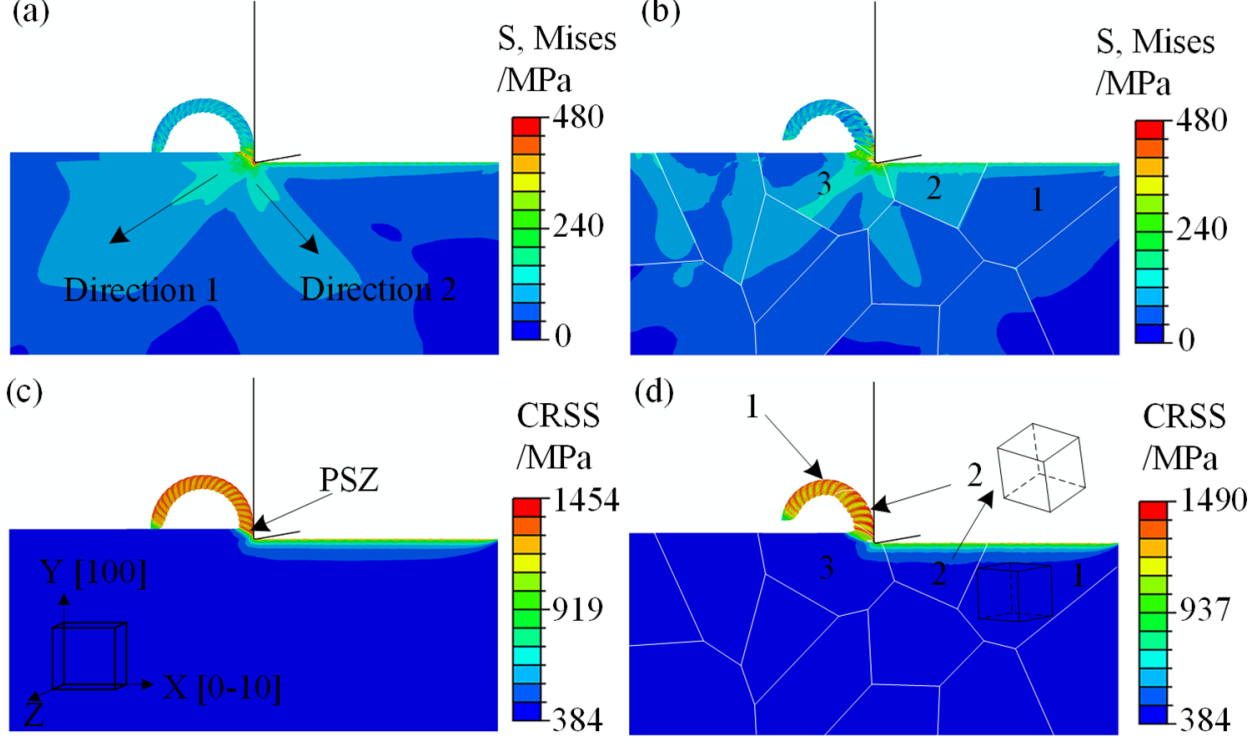
Fig. 10 Variations of machining results during FE simulations of diamond cutting of crystalline copper. Cutting force for a single crystalline copper and $\mathbf{b}$ polycrystalline copper; Surface height for c single crystalline copper and $\mathbf{d}$ polycrystalline copper
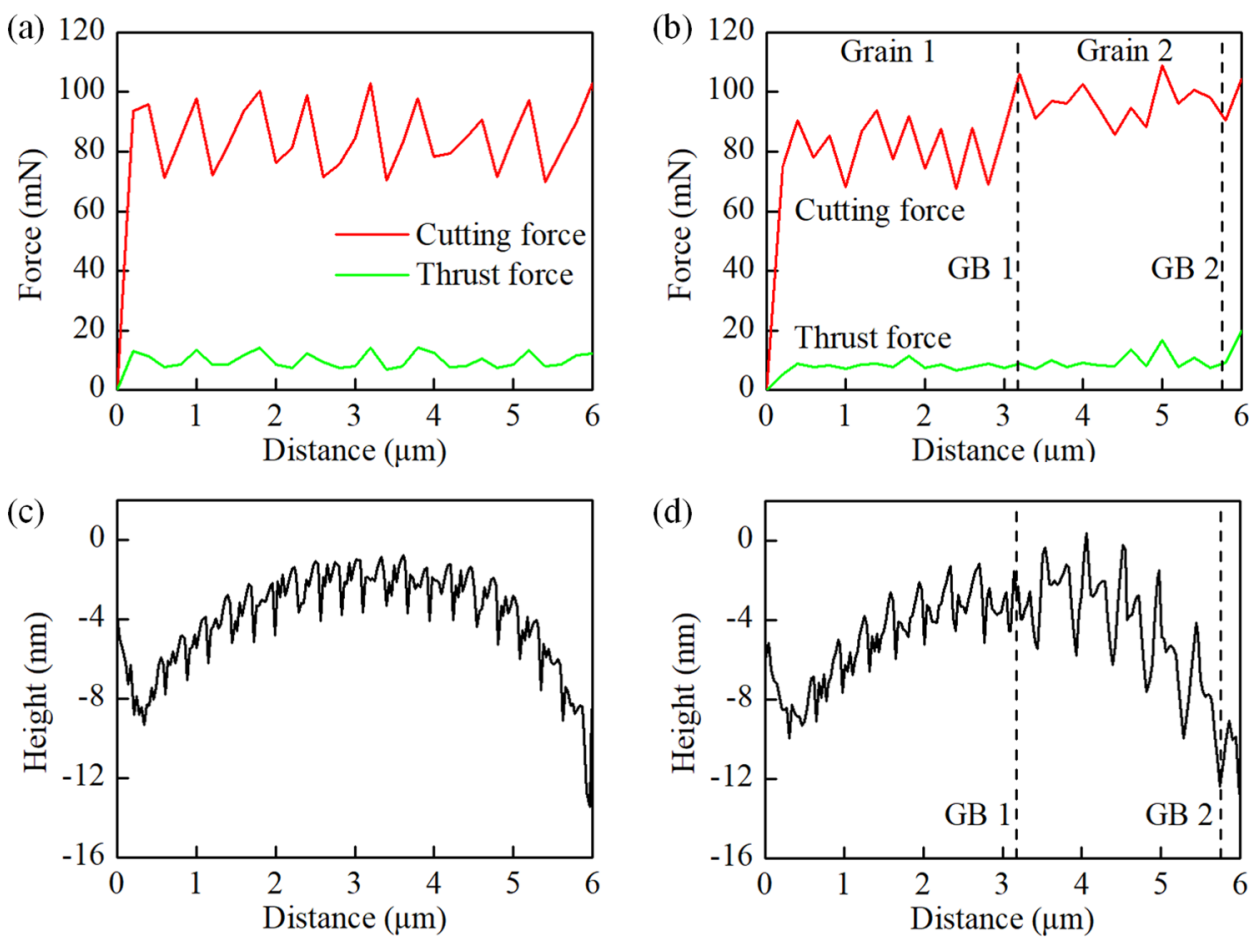

local uniformity within individual grain interior but a longrange anisotropy over individual grains. Furthermore, the chips formed in the cutting of polycrystalline copper are composed of serrations of complex lamella structures, in contrast to the long continuous chip obtained in cutting of single crystalline copper. The experimental finds above are validated by the prediction results from CPEF simulations. CPFE simulations based on stress analysis further reveal that the anisotropy influence is closely associated with the different degrees of elastoplastic deformation and recovery in individual grains, as well as the role of GBs playing in the confinement and initiation of plasticity.

Acknowledgements The authors greatly acknowledge support from the Science Challenge Project (Nos. TZ2018006-0201-02 and TZ2018006-0205-02) and the Fundamental Research Funds for the Central Universities.

Open Access This article is licensed under a Creative Commons Attribution 4.0 International License, which permits use, sharing, adaptation, distribution and reproduction in any medium or format, as long as you give appropriate credit to the original author(s) and the source, provide a link to the Creative Commons licence, and indicate if changes were made. The images or other third party material in this article are included in the article's Creative Commons licence, unless indicated otherwise in a credit line to the material. If material is not included in the article's Creative Commons licence and your intended use is not permitted by statutory regulation or exceeds the permitted use, you will need to obtain permission directly from the copyright holder. To view a copy of this licence, visit http://creativecommons.org/licenses/by/4.0/.

\section{References}

1. Hagen C, Hognestad A, Knudsen OØ, Sørby K (2019) The effect of surface roughness on corrosion resistance of machined and epoxy coated steel. Prog Org Coat 130:17-23

2. Fang FZ, Zhang N, Guo DM, Ehmann K, Cheung B, Liu K, Yamamura K (2019) Towards atomic and close-to-atomic scale manufacturing. Int J Extreme Manuf 1(1):12001

3. Fang FZ, Zhang XD, Weckenmann A, Zhang GX, Evans C (2013) Manufacturing and measurement of freeform optics. CIRP Ann 62(2):823-846

4. Rossi S, Deflorian F, Venturini F (2004) Improvement of surface finishing and corrosion resistance of prototypes produced by direct metal laser sintering. J Mater Process Technol 148(3):301-309

5. Brinksmeier E, Gläbe R, Osmer J (2006) Ultra-precision diamond cutting of steel molds. CIRP Ann-Manuf Technol 55(1):551-554

6. Zhang JG, Suzuki N, Wang YL, Shamoto E (2015) Ultra-precision nano-structure fabrication by amplitude control sculpturing method in elliptical vibration cutting. Precis Eng 39:86-99

7. Kong MC, Lee WB, Cheung CF, To S (2006) A study of materials swelling and recovery in single-point diamond turning of ductile materials. J Mater Process Technol 180(1-3):210-215

8. Zhang JJ, Shuai MB, Zheng HB, Li Y, Jin M, Sun T (2018) Atomistic and experimental investigation of the effect of depth of cut on diamond cutting of Cerium. Micromachines 9(1):26

9. Morris JC, Callahan DL, Kulik J, Patten JA, Scattergood RO (1995) Origins of the ductile regime in single-point diamond turning of semiconductors. J Am Ceram Soc 78(8):2015-2020

10. Zhang JG, Zhang JJ, Cui T, Hao ZW, Al Zahrani A (2017) Sculpturing of single crystal silicon microstructures by elliptical vibration cutting. J Manuf Process 29:389-398

11. Yan JW, Syoji K, Tamaki J (2003) Some observations on the wear of diamond tools in ultra-precision cutting of single-crystal silicon. Wear 255(7-12):1380-1387 
12. Nakajima T, Uno Y, Fujiwara T (1989) Cutting mechanism of fine ceramics with a single-point diamond. Precis Eng 11(1):19-25

13. Zhang JJ, Han L, Zhang JG, Liu HY, Yan YD, Sun T (2019) Brittle-to-ductile transition in elliptical vibration-assisted diamond cutting of reaction-bonded silicon carbide. J Manuf Process 45:670-681

14. Zhang JG, Suzuki N, Wang YL, Shamoto E (2014) Fundamental investigation of ultra-precision ductile machining of tungsten carbide by applying elliptical vibration cutting with single crystal diamond. J Mater Process Technol 214(11):2644-2659

15. Yan JW, Zhang ZY, Kuriyagawa T (2009) Mechanism for material removal in diamond turning of reaction-bonded silicon carbide. Int J Mach Tool Manuf 49(5):366-374

16. Yue XB, Xu M, Du WH, Chu C (2017) Effect of cutting edge radius on surface roughness in diamond tool turning of transparent MgAl2O4 spinel ceramic. Opt Mater 71:129-135

17. To S, Lee WB, Chan CY (1997) Ultraprecision diamond turning of aluminium single crystals. J Mater Process Technol 63(1-3):157-162

18. Zhou M, Ngoi B (2001) Effect of tool and workpiece anisotropy on microcutting processes. Proc Inst Mech Eng B-J Eng 215(1):13-19

19. Furukawa Y, Moronuki N (1988) Effect of material properties on ultra-precise cutting processes. CIRP Ann 37(1):113-116

20. Brinksmeier E, Schmütz J (1997) Generation and texture of surfaces in ultraprecision cutting of copper. Mach Sci Technol 1(2): 185-193

21. Yu JC, Wang G, Rong YM (2015) Experimental study on the surface integrity and chip formation in the micro cutting process. Procedia Manuf 1:655-662

22. Wang ZF, Zhang JJ, Zhang JG, Li G, Zhang HJ, Ul Hassan H, Hartmaier A, Yan YD, Sun T (2020) Towards an understanding of grain boundary step in diamond cutting of polycrystalline copper. J Mater Process Technol 276:116400

23. Wang ZF, Sun T, Zhang HJ, Li G, Li ZQ, Zhang JJ, Yan YD, Hartmaier A (2019) The interaction between grain boundary and tool geometry in nanocutting of a bi-crystal copper. Int J Extreme Manuf 1(4):045001
24. Fang FZ, Venkatesh VC (1998) Diamond cutting of silicon with nanometric finish. CIRP Ann-Manuf Technol 47(1):45-49

25. Fang FZ, Wu H, Zhou W, Hu XT (2007) A study on mechanism of nano-cutting single crystal silicon. J Mater Process Technol 184(1-3):407-410

26. Michler J, Rabe R, Bucaille J, Moser B, Schwaller P, Breguet J (2005) Investigation of wear mechanisms through in situ observation during microscratching inside the scanning electron microscope. Wear 259(1-6):18-26

27. Liu B, Xu ZW, Chen C, Li R, Wang CH, Yang X (2019) In situ experimental study on material removal behaviour of single-crystal silicon in nanocutting. Int J Mech Sci 152:378-383

28. Wang ZF, Zhang JJ, Xu ZW, Zhang JG, Ul Hassan H, Li G, Zhang HJ, Hartmaier A, Fang FZ, Yan YD, Sun T (2019) Crystal plasticity finite element modeling and simulation of diamond cutting of polycrystalline copper. J Manuf Process 38:187-195

29. Lee WB, To S, Sze YK, Cheung CF (2003) Effect of material anisotropy on shear angle prediction in metal cutting-mesoplasticity approach. Int J Mech Sci 45(10):1739-1749

30. Liu Q, Roy A, Tamura S, Matsumura T, Silberschmidt VV (2016) Micro-cutting of single-crystal metal: finite-element analysis of deformation and material removal. Int J Mech Sci 118:135-143

31. Wang ZF, Zhang JJ, Xu ZW, Zhang JG, Li G, Zhang HJ, Li ZQ, Ul Hassan H, Fang FZ, Hartmaier A, Yan YD, Sun T (2020) Crystal anisotropy-dependent shear angle variation in orthogonal cutting of single crystalline copper. Precis Eng 63:41-48

32. Fang FZ, Liu B, Xu ZW (2015) Nanometric cutting in a scanning electron microscope. Precis Eng 41:145-152

33. Quey R, Dawson PR, Barbe F (2011) Large-scale 3D random polycrystals for the finite element method: generation, meshing and remeshing. Comput Method Appl M 200(17-20):1729-1745

34. Wang ZF, Zhang JJ, Ul Hassan H, Zhang JG, Yan YD, Hartmaier A, Sun T (2018) Coupled effect of crystallographic orientation and indenter geometry on nanoindentation of single crystalline copper. Int J Mech Sci 148:531-539

35. Pan J, Rice JR (1983) Rate sensitivity of plastic flow and implications for yield-surface vertices. Int J Solids Struct 19:973-987 